\title{
A novel oligopeptide-simulating dentine matrix protein 1 for biomimetic mineralization of dentine
}

\section{Ying Cao ${ }^{a}$, Wei Liu ${ }^{b}$, Tianyun Ning ${ }^{b}$, May L. Mei ${ }^{a}$, C. H. Chu ${ }^{\text {a }}$, Quan- Li Li ${ }^{b}$, Edward C. M. Lo ${ }^{a}$}

${ }^{a}$ Faculty of Dentistry, The University of Hong Kong, Hong Kong SAR, China

${ }^{\mathrm{b}}$ College of Stomatology, Anhui Medical University, Hefei, P R China

Key words: Biomineralization, Dentine Matrix Protein, Oligopeptide, Biomimetic Remineralization, Collagen, Hydroxyapatite

\author{
Corresponding to : $\quad{ }^{a} \mathrm{C} . \mathrm{H}$. Chu \\ Faculty of Dentistry, \\ The University of Hong Kong, \\ 34 Hospital Road, Hong Kong SAR, China. \\ Tel: +852 2859-0287 \\ Fax: +852 2858-7874 \\ Email: chchu@hku.hk \\ ${ }^{\mathrm{b}}$ Quan-Li Li \\ College of Stomatology, \\ Anhui Medical University, \\ 69 Meishan Road, Hefei, P R China \\ Tel (Tax): +86 551-5172125 \\ Email: q1-li@126.com
}




\begin{abstract}
Objective: The objectives were to design and fabricate an oligopeptide that simulates dentine matrix protein 1 (DMP1) to study its ability to bind to dentine collagen fibrils and induce biomimetic mineralization for the management of dentine hypersensitivity.
\end{abstract}

Materials and Methods: A novel oligopeptide was developed by connecting the collagen-binding domain of DMP1 to the hydrophilic C-terminal of amelogenin. Fluorescein isothiocyanate-coupled oligopeptide was applied to the completely demineralized dentine collagen and examined using fluorescent microscopy. The nucleation and growth of hydroxyapatite was initiated by immersing oligopeptide into calcium chloride and sodium hydrogen phosphate solutions. Scanning electron microscopy (SEM), transmission electron microscopy (TEM), and selected area electron diffraction (SAED) were used to examine the formation. Dentine slices were acid-etched, coated with oligopeptide, and immersed into a metastable calcium phosphate solution. Dentine mineralization was evaluated by SEM, X-ray diffraction (XRD), and Fourier transform infrared spectroscopy (FTIR).

Results: Fluorescent dentine collagen was identified in the specimens. The nucleation and growth of crystals were detected after immersing the oligopeptide into calcium chloride and sodium hydrogen phosphate solutions. Under SEM, crystals were observed covering the oligopeptide-coated dentine surface, within the demineralized dentine collagen matrix, and occluding dentinal tubules. SAED, XRD and FTIR confirmed that the crystals were hydroxyapatite.

Conclusion: A novel oligopeptide-simulating DMP1 was developed that can bind to collagen fibrils, initiate mineralization, and induce biomimetic mineralization of dentine. Clinical Relevance. Biomimetic mineralization of dentine facilitated by this oligopeptide is a potential therapeutic technique for the management of dentine hypersensitivity. 


\section{Introduction}

Gingival recession due to periodontal disease and tooth brushing abrasion can result in the subsequent exposure of root surfaces to hypersensitivity [1]. The exposed root surface is also susceptible to root caries, which are often notoriously difficult to restore. Various treatments have been proposed to treat hypersensitivity, such as bonding agents, desensitizing toothpaste, fluoride varnish, laser therapy, and restoration. However, these treatments are sometimes unsuccessful. Further, even when they work, hypersensitivity frequently recurs. Novel strategies, such as biomimetic mineralization, need to be developed to manage this increasingly common problem.

Biomineralization is an organic matrix-mediated biomineralization process [2]. Tarasevich et al [3] successfully demonstrated the nucleation and growth of calcium phosphate crystals by amelogenin on an enamel surface. In clinical dentistry, the most commonly used remineralization agents are supersaturated solutions of calcium and phosphate and fluoride solutions or gels [4]. Although these solutions and gels can promote enamel remineralization and reduce the number of dental caries, they have little effect on dentine remineralization $[5,6]$.

Enamel is highly mineralized with approximately $97 \%$ by weight hydroxyapatite (HAP). The HAP nano-crystals self-assemble into a special microstructure of repeating prism units. In the presence of a supersaturated calcium phosphate solution, the HAP nano-crystals can act as nuclei to induce the growth of HAP crystals, thereby resulting in the precipitation of HAP crystals on the enamel surface [7]. To become more resistant to caries attack than the original enamel tissue, the HAP nano-crystals precipitate on the surface and penetrate the subsurface areas of the carious lesion. Remineralization of carious lesions in enamel is different from that of dentine, which contains a substantial amount of collagen. The aim of the remineralization of dentine is to form the precipitation of minerals onto the dentine surface and within the demineralized dentine collagen matrix [2]. In addition, it is advantageous to occlude the open dentinal tubules for the 
management of dentine hypersensitivity and dental erosion. The process of dentine remineralization differs from that of enamel remineralization due to the differences in their compositions and structures. By weight, dentine is comprised of 70\% HAP, 20\% organic matrix that primarily consists of collagen, and $10 \%$ water. Its structure is characterized by a calcified collagen matrix and dentinal tubules. After demineralization, the collagen matrix is exposed on the dentine surface. However, it is ineffective in initializing HAP nucleation and growth. Results of previous studies have shown that precipitation of HAP crystals is rare and tends to be unevenly distributed on the dentine surface in the presence of a remineralizing solution $[6,8]$.

The organic matrix of dentine mainly consists of type I collagen [9], which can act as a template and plays an important role in the biomineralization of hard tissues, such as bone and dentine. Nevertheless, type I collagen is inefficient as a structural matrix for inducing matrix-specific mineral formation [10]. Non-collagenous proteins (NCPs), which are tightly bound to the collagen fibers in mineralized tissues, are required as the functional matrix for depositing HAP on the collagen matrix. NCPs, such as dentine, DMP1, and dentine phosphophoryn, bind to collagen fibrils and contribute to the regulation (initiation or inhibition) of dentine biomineralization [11]. DMP1 is the main acidic non-collagenous matrix phosphoprotein identified from the mineralized matrix of dentine and bone $[12,13]$. It strongly influences collagen mineralization and regulates HAP nucleation and growth [14-17]. DMP1 is also essential for dentinogenesis. Ye et al [18] reported that DMP1-null mice postnatally developed a distinct tooth phenotype characterized by the partial failure of predentine to mature into dentine, enlarged pulp chambers, increased width of the predentine zone with a reduced dentine wall, and hypomineralization.

Some researchers have used DMP1 to mimic enamel and dentine biomineralization processes $[16,19]$. DMP1 is a natural protein that is difficult to extract and purify, as it can easily denature and is subject to potential contamination. However, synthetic oligopeptide can be produced using self-assembling short peptides. The aim of 
this study was to design and fabricate a synthetic oligopeptide stimulating the function of DMP1 that induces the nucleation and growth of HAP for dentine mineralization. The null hypotheses were: (1) the synthetic oligopeptide cannot bind to collagen, (2) the synthetic oligopeptide cannot induce the nucleation and growth of HAP, and (3) the synthetic oligopeptide cannot induce dentine mineralization.

\section{Materials and Methods}

\section{Synthesis of the oligopeptide simulating dentine matrix protein 1}

An oligopeptide with an amino acid sequence of "Asp-Ser-Glu-Ser-Ser-GluGlu-Asp-Arg-Thr-Lys-Arg-Glu-Glu-Val-Asp" was produced by connecting the collagen-binding domain of DMP1 (Asp-Ser-Glu-Ser-Ser-Glu-Glu-Asp-Arg-) to the hydrophilic C-terminal (-Thr-Lys-Arg-Glu-Glu-Val-Asp) of amelogenin. It was synthesized by standard solid-phase peptide synthesis (SPPS) using standard fluorenylmethoxycarbonyl (Fmoc) chemistry on an Applied Biosystems 433A automated peptide synthesizer [20]. The SPPS was based on the sequential addition of alpha-amino and side-chain protected amino acid residues. Various amino acids were connected oneby-one via amide bonds. The base-labile Fmoc-group was used for $\mathrm{N}$-alpha-protection. After removal of this protecting group, the next protected amino acid was added using a pre-activated, protected amino acid derivative. The resulting peptide was attached to the resin via a linker through its $\mathrm{C}$-terminus. Side-chain protected groups were selected to be cleaved simultaneously to the detachment of the peptide from the resin. The synthetic oligopeptide was purified and characterized by high-performance liquid chromatography (HPLC) and mass spectrometry. Deionized water was used to dissolve the oligopeptide to form a solution with concentrations of $1 \mathrm{mg} / \mathrm{ml}$ and $10 \mu \mathrm{g} / \mathrm{ml}$ for subsequent studies.

\section{Ability to bind to dentine collagen}

The ability of the oligopeptide to bind to dentine collagen was assessed using a fluorescent staining procedure. Fluorescein isothiocyanate (FITC) is a fluorescent staining dye derived from fluorescein. An FITC-coupled oligopeptide was also 
synthesized by binding the $\mathrm{N}$-terminus of the oligopeptide to carboxylic groups of the FITC protein using SPPS. The FITC-coupled oligopeptide was used in the fluorescent staining test to confirm that the oligopeptide specifically bound to dentine collagen. Dentine slices that were $1.5 \mathrm{~mm}$ thick were prepared from extracted sound human teeth. The slices were completely demineralized with $0.5 \mathrm{M}$ of EDTA $(\mathrm{pH}=8)$ for 5 days (with stirring) at $25^{\circ} \mathrm{C}$ and washed with deionized water 3 times. They were then treated with $4 \mathrm{M}$ guanidine $\mathrm{HCl}$ overnight at $4^{\circ} \mathrm{C}$ for the extraction of phosphoprotein and $0.5 \mathrm{M}$ $\mathrm{NaCl}, 50 \mathrm{mM}$ Tris- $\mathrm{HCl}$ solution $(\mathrm{pH}=7.4)$. One slice was used for the energy dispersive X-ray detector to verify the complete demineralization of the dentine slices. Another slice was cut into a rectangular demineralized dentine block. The length and width of the block was measured using a digital micrometer. The block was then sectioned into 10$\mu \mathrm{m}$ thick specimens using cryostat microtome (CM1850, Leica Cryostat CMS GmbH, Wetzlar, Germany) in a cryochamber at $-20^{\circ} \mathrm{C}$.

Some of the $10-\mu \mathrm{m}$ thick demineralized dentine specimens were used in the subsequent experiment. They were fixed on glass slides and immersed in a $1 \%$ bovine serum albumin (BSA) solution for $30 \mathrm{~min}$ at $37^{\circ} \mathrm{C}$ to block non-special adsorption. The specimens were divided into three groups. In group $1,50 \mu \mathrm{l}$ of $0.1 \mathrm{mg} / \mathrm{ml} \mathrm{FITC-}$ coupled oligopeptide solution was applied to the specimen for fluorescent staining of the collagen. In group 2, $50 \mu \mathrm{l}$ of $0.1 \mathrm{mg} / \mathrm{ml}$ of FITC-labeled anti-IgG antibodies (Boster Biological Technology, Ltd. Wuhan, China) was applied to the specimens for nonspecial fluorescent protein adsorption staining. In group 3, a phosphate-buffered solution (PBS) was applied to the specimens for assessing the dentine's auto-fluorescence. The specimens were incubated at $37^{\circ} \mathrm{C}$ and $100 \%$ humidity in an aluminum foil box for 30 min and washed with a PBS for 5 min. After they were washed three times with the PBS, the specimens were observed using fluorescence microscopy for immunofluorescent staining (DMI 3000, Leica Microsystems CMS GmbH, Wetzlar, Germany).

In another experiment, $2410-\mu \mathrm{m}$ thick demineralized dentine specimens were placed in a sterilized 24-well tissue culture microplate and immersed in 1\% BSA solution 
for $30 \mathrm{~min}$ at $37^{\circ} \mathrm{C} .10 \mu \mathrm{l}$ of $0.1 \mathrm{mg} / \mathrm{ml}$ of FITC-coupled oligopeptide solution was applied to 12 specimens. In addition, $10 \mu \mathrm{l}$ of $0.1 \mathrm{mg} / \mathrm{ml}$ of FITC-labeled anti-IgG antibodies solution was applied to the remaining 12 specimens. The microplate was incubated at $37^{\circ} \mathrm{C}$ and $100 \%$ humidity in a lucifugal box for $30 \mathrm{~min}$. Further, 1,000 $\mathrm{ml}$ of PBS was added to the wells to dissolve the non-binding FITC-coupled oligopeptide and non-binding FITC-labeled anti-IgG antibodies. Also, $10 \mu \mathrm{l}$ of $0.1 \mathrm{mg} / \mathrm{ml}$ of FITCcoupled oligopeptide solution and $10 \mu \mathrm{l}$ of $0.1 \mathrm{mg} / \mathrm{ml}$ of FITC-labeled anti-IgG antibodies was added into 2 wells without a dentine slice containing 1,000 $\mathrm{ml}$ of PBS. A fluorometer was used to measure the concentration of FITC-coupled oligopeptide and FITC-labeled anti-IgG antibodies in the PBS. The quantity of FITC-coupled oligopeptide and FITC-labeled anti-IgG antibodies binding to dentine collagen was calculated. Moreover, the differences between the two groups were assessed using a twosample t-test.

\section{Ability to induce hydroxyapatite nucleation and growth}

The ability of the oligopeptide to induce HAP nucleation and grow th was examined using scanning electron microscopy (SEM) at x300,000 magnification (Sirion 200, FEI Co., Hillsboro, USA) and transmission electron microscopy (TEM) at x300,000 magnification (JEOL-2010, JEOL Ltd. Co., Tokyo, Japan). The synthetic oligopeptide solution $(1 \mathrm{mg} / \mathrm{ml})$ was applied on the TEM copper grids and dried at room temperature before fixation with $2 \%$ glutaraldehyde for $30 \mathrm{~min}$. The grids were immersed in $10 \mathrm{mM}$ of calcium chloride $\left(\mathrm{CaCl}_{2}\right)$ solution for $60 \mathrm{~min}$ before being immersed in $5 \mathrm{mM}$ of sodium hydrogen phosphate $\left(\mathrm{Na}_{2} \mathrm{HPO}_{4}\right)$ solution for $60 \mathrm{~min}$ at $23^{\circ} \mathrm{C}$. The procedure was repeated five times before examination by SEM and TEM. The grids without oligopeptide application were used as a control.

\section{Ability to induce dentine mineralization}

Dentine slices that were $2 \mathrm{~mm}$ thick were prepared and polished with an 800grit silicon carbide paper under running water, etched with $37 \%$ phosphoric acid for 1 min to expose the collagen [21], and rinsed with deionized water to remove the acid. The 
slices were then divided into an experimental and a control group. The slices in the experimental group were immersed in $0.1 \mathrm{mg} / \mathrm{ml}$ of oligopeptide solution for 24 hours, whereas those in the control group were immersed in deionized water. Each slice was placed in a sealed polyethylene tube filled with $10 \mathrm{ml}$ of biomimetic mineralization solution. The biomimetic mineralization solution contains $2.58 \mathrm{mM} \mathrm{Ca}^{2+}, 1.55 \mathrm{mM} \mathrm{PO}_{4}{ }^{3-}$, and 1 ppm $\mathrm{F}^{-}$buffered by $50 \mathrm{mM}, \mathrm{pH}=7.6$ Tris buffer, and $180 \mathrm{mM} \mathrm{NaCl}$ ). The slices were incubated at $37^{\circ} \mathrm{C}$. The biomimetic mineralization solution was replaced every 24 hours. The slices were dehydrated with ethanol and dried in the critical evaporator after 48 hours before SEM evaluation.

The ability of the oligopeptide to induce dentine mineralization was examined using SEM (Hitachi S-4800, Hitachi High Technologies America Inc., Dallas, USA; Sirion 200, FEI Co., Hillsboro, USA), X-ray diffraction (XRD) (X'Pert Pro, Philips, Almelo, The Netherlands), and diffuse reflectance-Fourier transform infrared spectroscopy (DR-FTIR) (Nicolet 8700, Thermo Scientific Instrument Co., New Hampshire, USA).

\section{Results}

\section{Design and synthesis of the oligopeptide}

The amino acid sequence of the oligopeptide was "Asp-Ser-Glu-Ser-Ser-Glu-GluAsp-Arg-Thr-Lys-Arg-Glu-Glu-Val-Asp” (Fig. 1). The purity of the obtained oligopeptide was $98 \%$ (Fig. 2). The mass spectrum of the purified oligopeptide showed a single peak corresponding to a molecular weight of $1911 \mathrm{Da}$, which was close to the theoretical molecular weight (Fig. 3).

\section{Binding to dentine collagen}

The completely demineralized dentine samples that were treated with FITCcoupled oligopeptide (group 1) showed extensive green immunofluorescent staining in the fluorescent micrographs, which confirmed the binding of the synthetic 
oligopeptide to the dentine collagen (Fig. 4a). No florescence was observed in the samples that were treated with FITC-labeled anti-IgG antibodies (group 2; Fig. 4b) or the phosphate-buffered solution (group 3; Fig 4c). Therefore, the special adsorption caused the oligopeptide to bind to the collagen.

The quantity of FITC-coupled oligopeptide that bound to dentine collagen $(0.2104 \pm 0.0016 \mu \mathrm{g})$ was significantly greater than that of FITC-labeled anti-IgG antibodies $(0.0583 \pm 0.0001 \mu \mathrm{g})(\mathrm{p}<0.001)$. Given that the surface area of the dentine block was measured as $5.431 \mathrm{~mm} \times 1.112 \mathrm{~mm}$, the quantity of FITC-coupled oligopeptide that bound to dentine collagen was approximately $0.0348 \mu \mathrm{g} / \mathrm{mm}^{2}$.

\section{Inducing HAP nucleation and growth}

The SEM images revealed the plate-like morphology of the HAP precipitation (Fig. 5a). The TEM images showed that the oligopeptide as a DMP1 simulator could capture calcium and phosphate ions to induce HAP nucleation and growth following alternate immersions in $\mathrm{CaCl}_{2}$ and $\mathrm{Na}_{2} \mathrm{HPO}_{4}$ solutions (Fig. 5b). Electron diffraction of these crystallites revealed discrete ring patterns, which are characteristic of HAP (Fig. 5b inset). No crystals were found in the control grids (Fig. 5c, d).

\section{Inducing dentine mineralization}

The XRD patterns of the HAP precipitates on the dentine surface are shown in Figure 6. The peaks at $2 \theta^{\circ}=25.968^{\circ}, 31.699^{\circ}, 32.079^{\circ}$, and $32.782^{\circ}$ correspond to the expected HAP peaks at the $002,211,112$, and 300 faces, respectively, thereby confirming that the precipitates were HAP crystals. The peaks ascribed to the 211,112 , and 300 faces were not completely separated, suggesting that the HAP precipitates that formed were low in crystallinity. The 002 face had a much stronger peak than those of the 112,211 , and 300 faces, suggesting that the HAP crystals precipitated along the c-axis.

Figure 7 represents the DR-FTIR spectra generated from the oligopeptide-coated dentine collagen surfaces and acid-etched dentine surface without oligopeptide coating 
after immersion in the biomimetic mineralization solution for 48 hours. The FTIR spectra indicated the presence of protein and phosphate minerals on the oligopeptide-coated dentine collagen surface. The split $v_{4} \mathrm{PO}_{4}$ peaks at approximately 556 and $607 \mathrm{~cm}^{-1}$ and $v_{1} \mathrm{PO}_{4}$ peaks at approximately $1124 \mathrm{~cm}^{-1}$ were from HAP crystals. The amide I (1600$\left.1690 \mathrm{~cm}^{-1}\right)$ and amide II $\left(1480-1575 \mathrm{~cm}^{-1}\right)$ bands were derived from the $\mathrm{C}=\mathrm{O}$ and $\mathrm{NH}$ stretch vibrations from the exposed dentine collagen [22]. The amide III band (1229-1301 $\mathrm{cm}^{-1}$ ) along line (a) was due to the $\mathrm{CN}$ stretch vibrations, which probably originated from the oligopeptide links.

Following 48-hour immersion in the metastable calcium phosphate solution, HAP precipitation was found covering the dentine surface and occluding all dentinal tubules on the oligopeptide-coated dentine collagen (Fig. 8a, b). However, it was not found on the control group collagen (Fig. 9). In the transverse section of the oligopeptide coating samples, HAP crystals that precipitated in the dentinal tubules extended far from the demineralized areas (Fig. 8b). The HAP crystals precipitated in the demineralized collagen matrix and remineralized the peritubular dentine (Fig. 8c, d). Further, HAP crystals grew along the dentinal tubules and packed together to occlude the dentinal tubules (Fig. 8e, f).

\section{Discussion}

The null hypotheses were rejected. Results showed that the synthetic oligopeptide could bind to the collagen, suggesting the nucleation and growth of HAP. Findings also showed that the HAP that precipitated on the dentine surface occluded the openings of the dentinal tubules and remained attached to them following supersonic treatment. The oligopeptide could be used as a potential therapeutic agent to treat dentine hypersensitivity.

The fabricated novel oligopeptide copied the amino acid sequence of the collagenbinding domain of DMP1 “Asp-Ser-Glu-Ser-Ser-Glu-Glu-Asp-Arg” [23] and the amino 
acid sequence (-Thr-Lys-Arg-Glu-Glu-Val-Asp) of the hydrophilic C-terminal of amelogenin. Amelogenin, an enamel protein, constitutes approximately $90 \%$ of the organic matrix in the enamel of human teeth. It can spontaneously self-assemble into a nanospherical supra-molecular structure, which plays a vital role in enamel prism formation [3]. The self-assembly mechanism of amelogenin is attributed to its bipolar structure with the hydrophilic C-terminal (-Thr-Lys-Arg-Glu-Glu-Val-Asp) on the globular monomer surface. The C-terminal is able to interact with calcium ions and initializes HAP nucleation. By connecting the hydrophilic C-terminal of amelogenin to the collagen-binding domain of DMP1, this study successfully fabricated a novel oligopeptide that was able to bind to collagen and occlude dentinal tubules for the management of hypersensitivity.

Similar to the synthesis of other synthetic peptides, the novel oligopeptide created in this study was fabricated by coupling the carboxyl group or C-terminus of one amino acid to the amino group or N-terminus of another amino acid. This method of synthesis differs from ribosome protein synthesis. The classical solution-phase peptide synthesis method was not used in this study because the oligopeptide has a fairly long sequence. The repetition of coupling and de-protection cycles is labor-intensive and requires the isolation of all peptide intermediates. SPPS is more efficient than solutionphase peptide synthesis, as the purification procedures are simpler to perform. However, SPPS has limited yields, which can be a problem for the synthesis of long-chain peptides. This study successfully demonstrated the SPPS protocol because the fabricated oligopeptide could be purified to $98 \%$.

The remineralization of enamel and dentine is often a more favorable option than restorative treatment in the management of erosion. The remineralization of dentine is more difficult than that of enamel because of its composition and structure. However, clinicians commonly encounter severe erosive lesions involving dentine. The demineralized dentine surface caused by dental erosion is composed primarily of a crosslinking collagen matrix, which is ineffective for inducing HAP nucleation and growth. 
An initiator is essential for crystal nucleation and growth [24].

Researchers have proposed the use of poly-vinyl phosphoric acid [25-27], sodium trimetaphosphate [28], and tripolyphosphate [29] to modify collagen protein. These methods introduce phosphate groups into collagen molecules to simulate the role of NCPs in initiating biomimetic dentine/collagen matrix mineralization. However, the binding of phosphate groups to collagen molecules is random. Researchers have also used DMP1 to induce dentine/collagen matrix remineralization [14-16]. DMP1 is a natural NCP that has a high affinity for HAP and plays an important role in initiating and regulating HAP nucleation and growth. It binds to collagen at the site of the gap zone of the collagen fibrils and facilitates segmentally remineralized collagen fibers [14]. It also binds to mineral ions and stabilizes mineral precursors to control collagen mineralization. Thus, the aim of this study was to design and fabricate an oligopeptide to simulate DMP1 for the biomimetic mineralization of dentine.

To simulate DMP1, the oligopeptide needed to be able to bind to collagen proteins and calcium ions to induce HAP nucleation. In addition, it needed to be able to induce collagen matrix mineralization. Using the immunofluorescent staining technique, we demonstrated that the oligopeptide could bind to collagen. When the TEM copper grid was coated with oligopeptide and subjected to repeated cycles of immersion in $\mathrm{CaCl}_{2}$ and $\mathrm{Na}_{2} \mathrm{HPO}_{4}$ solutions, it was found that the oligopeptide could capture calcium and phosphate ions to induce HAP nucleation and growth.

Phosphoric acid was used to demineralize the dentine surface because it is a commonly used acid-etching agent in clinical dentistry. Milder acidic solutions or an EDTA solution could have been used for demineralization. The dentine collagen, which was exposed by demineralization, could then be exposed to the synthetic oligopeptide and metastable calcium phosphate solution. Using SEM, XRD, and FTIR, HAP precipitation was found on the dentine surface. HAP was also precipitated within the demineralized collagen matrix and occluded dentinal tubules. The results suggested that the oligopeptide 
could induce dentine remineralization. Further study using a bovine collagen membrane can be performed to demonstrate the ability of this oligopeptide to mineralize collagen fibrils [30]. Although XRD also revealed HAP in the control group, it was in the dentine under the demineralized layer through which the X-ray penetrated.

Lui et al [31] classified the fabrication of nano-biomaterials into "top-down" and "bottom-up" approaches. In a "top-down" approach, a complex entity is broken down into its component parts to assemble a biomaterial. In a "bottom-up" approach, a biomaterial is assembled from nano-sized molecules. A metastable calcium phosphate solution was used as the mineral ion source, indicating a "top-down" mineralization strategy [31]. It has been reported that the "top-down" method cannot induce intrafibrillar apatite formation [31]. However, this study found precipitation of HAP crystals within the collagen matrix. It may be that the peptide that bonded to the collagen fibers captured calcium ions to form amorphous mineral precursors of HAP.

Biomineralization is a "bottom-up" approach that involves the spatial regulation of amorphous mineral phases via matrix proteins and organization [31]. The addition of an organic matrix to the mineralization solution could induce amorphous precursors that are small enough to penetrate a demineralized collagen matrix [21]. An additional study is being conducted to explore the possibility of employing a "bottom-up" mineralization strategy, such as using a stable nano-ACP precursor combined with the DMP1-like oligopeptide to induce intrafibrillar mineralization to regenerate the dentine microstructure.

\section{Conclusion}

A novel synthetic oligopeptide that could bind to dentine collagen and induce the nucleation and growth of hydroxyapatite on the acid-etched dentine surface and along the dentinal tubules was successfully designed and fabricated. As this synthetic oligopeptide can facilitate biomimetic mineralization and occlude dentinal tubules, it 
offers a potential molecular tool for the management of dentine hypersensitivity.

\section{Acknowledgement}

This study was supported by grants from the NSFC/RGC Joint Research Scheme (N_HKU 776/10 and No.81061160511), NSFC (No.30973352), Outstanding Youth Fund of the Board of Education of Anhui Province (No.2011SQRL063), and Youth Foundation of Anhui Provincial Natural Science Foundation (No.1208085QH144).

\section{Conflicts of interest}

The authors declare that they have no conflicts of interest and/or any financial interests in any of the used products. 


\section{References}

1. Chu CH, Lam A, Lo EC (2011) Dentin hypersensitivity and its management. Gen Dent 59: 115-122. doi

2. Ning TY et al (2012) Biomimetic mineralization of dentin induced by agarose gel loaded with calcium phosphate. J Biomed Mater Res B Appl Biomater 100: 138-144.

3. Tarasevich BJ et al (2007) The nucleation and growth of calcium phosphate by amelogenin. J Cryst Growth 304: 407-415.

4. Chu CH, Mei ML, Lo EC (2010) Use of fluorides in dental caries management. Gen Dent 58: 37-43.

5. Saito T, Yamauchi M, Crenshaw MA (1998) Apatite induction by insoluble dentin collagen. J Bone Miner Res 13: 265-270.

6. Kawasaki K et al. (1999) The remineralization of EDTA-treated human dentine. Caries Res 33: 275-280.

7. Luo JJ et al (2012) Biomimic enamel remineralization by hybridization calcium- and phosphate-loaded liposomes with amelogenin-inspired peptide. High-Performance Ceramics 512-515: 1727-1730.

8. Ten Cate JM (2001) Remineralization of caries lesions extending into dentin. J Dent Res 80: 1407-1411.

9. Mei ML et al (2012) The inhibitory effects of silver diamine fluoride at different concentrations on matrix metalloproteinases. Dental Materials 28: 903-908.

10. Zhou YZ et al (2012) Polydopamine-induced tooth remineralization. ACS Appl Mater Interfaces 4: 6901-6910.

11. George A, Veis A(2008) Phosphorylated proteins and control over apatite nucleation, crystal growth, and inhibition. Chem Rev 108: 4670-4693.

12. George A et al (1993) Characterization of a novel dentin matrix acidic phosphoprotein. Implications for induction of biomineralization. J Biol Chem 268: 12624-12630.

13. MacDougall $\mathrm{M}$ et al (1998) Identification of a novel isoform of mouse dentin matrix protein 1: spatial expression in mineralized tissues. J Bone Miner Res 13: 422-431. 
14. He G, George A (2004) Dentin matrix protein 1 immobilized on type I collagen fibrils facilitates apatite deposition in vitro. J Biol Chem 279: 11649-11656.

15. Tartaix PH et al (2004) In vitro effects of dentin matrix protein-1 on hydroxyapatite formation provide insights into in vivo functions. J Biol Chem 279: 18115-18120.

16. He G et al (2003) Nucleation of apatite crystals in vitro by self-assembled dentin matrix protein 1. Nat Mater 2: 552-558.

17. He G et al (2005) Spatially and temporally controlled biomineralization is facilitated by interaction between self-assembled dentin matrix protein 1 and calcium phosphate nuclei in solution. Biochemistry 44: 16140-16148.

18. Ye L et al (2004) Deletion of dentin matrix protein-1 leads to a partial failure of maturation of predentin into dentin, hypomineralization, and expanded cavities of pulp and root canal during postnatal tooth development. J Biol Chem 279: 1914119148.

19. He $\mathrm{G}$ et al (2003) Dentin matrix protein 1 initiates hydroxyapatite formation in vitro. Connect Tissue Res 44 Suppl 1: 240-245.

20. Cao Y, Mei ML, Xu J, Lo ECM, Li QL, Chu CH. (in press) Biomimetic mineralisation of phosphorylated dentine by CPP-ACP. J Dent.

21. Tay FR, Pashley DH (2008) Guided tissue remineralisation of partially demineralised human dentine. Biomaterials 29: 1127-1137.

22. Kong J, Yu S (2007) Fourier transform infrared spectroscopic analysis of protein secondary structures. Acta Biochim Biophys Sin (Shanghai) 39: 549-559.

23. He $\mathrm{G}$ et al (2003) Nucleation of apatite crystals in vitro by self-assembled dentin matrix protein, 1. Nature Materials 2: 552-558.

24. Saito T et al (1997) Mineral induction by immobilized phosphoproteins. Bone 21: 305-311.

25. Gu LS et al (2011) Immobilization of a phosphonated analog of matrix phosphoproteins within cross-linked collagen as a templating mechanism for biomimetic mineralization. Acta Biomater 7: 268-277.

26. Liu $\mathrm{Y}$ et al (2011) Intrafibrillar collagen mineralization produced by biomimetic hierarchical nanoapatite assembly. Adv Mater 23: 975-980. 
27. Thula TT et al (2011) In vitro mineralization of dense collagen substrates: a biomimetic approach toward the development of bone-graft materials. Acta Biomater 7: $3158-3169$.

28. Gu LS et al (2010) A chemical phosphorylation-inspired design for Type I collagen biomimetic remineralization. Dent Mater 26: 1077-1089.

29. Xu Z, Neoh KG, Kishen A (2010) A biomimetic strategy to form calcium phosphate crystals on type I collagen substrate. Materials Science \& Engineering C-Materials for Biological Applications 30: 822-826.

30. Kim J et al (2010) Functional biomimetic analogs help remineralize apatite-depleted demineralized resin-infiltrated dentin via a bottom-up approach. Acta Biomaterialia 6: $2740-2750$.

31. Liu Y et al (2011) Differences between top-down and bottom-up approaches in mineralizing thick, partially demineralized collagen scaffolds. Acta Biomater 7: 1742-1751. 
Fig. 1 Framework of the synthetic oligopeptide-simulating dentine matrix protein 1 (a) amino acid sequence and (b) space-filling model
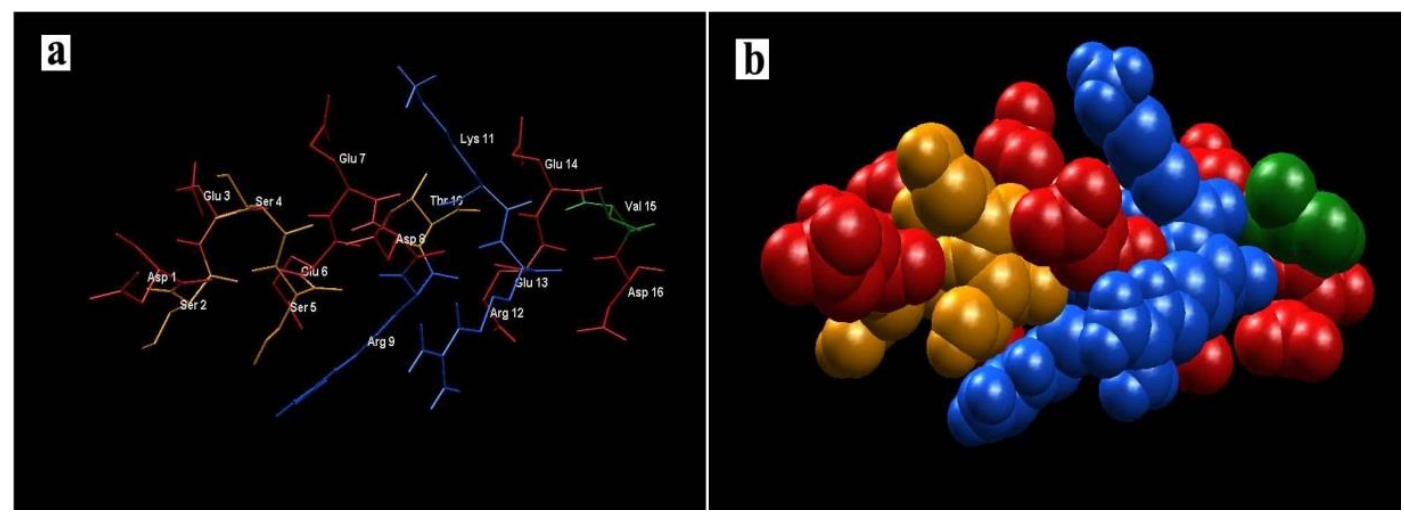

Fig. 2 HPLC report of the synthetic oligopeptide

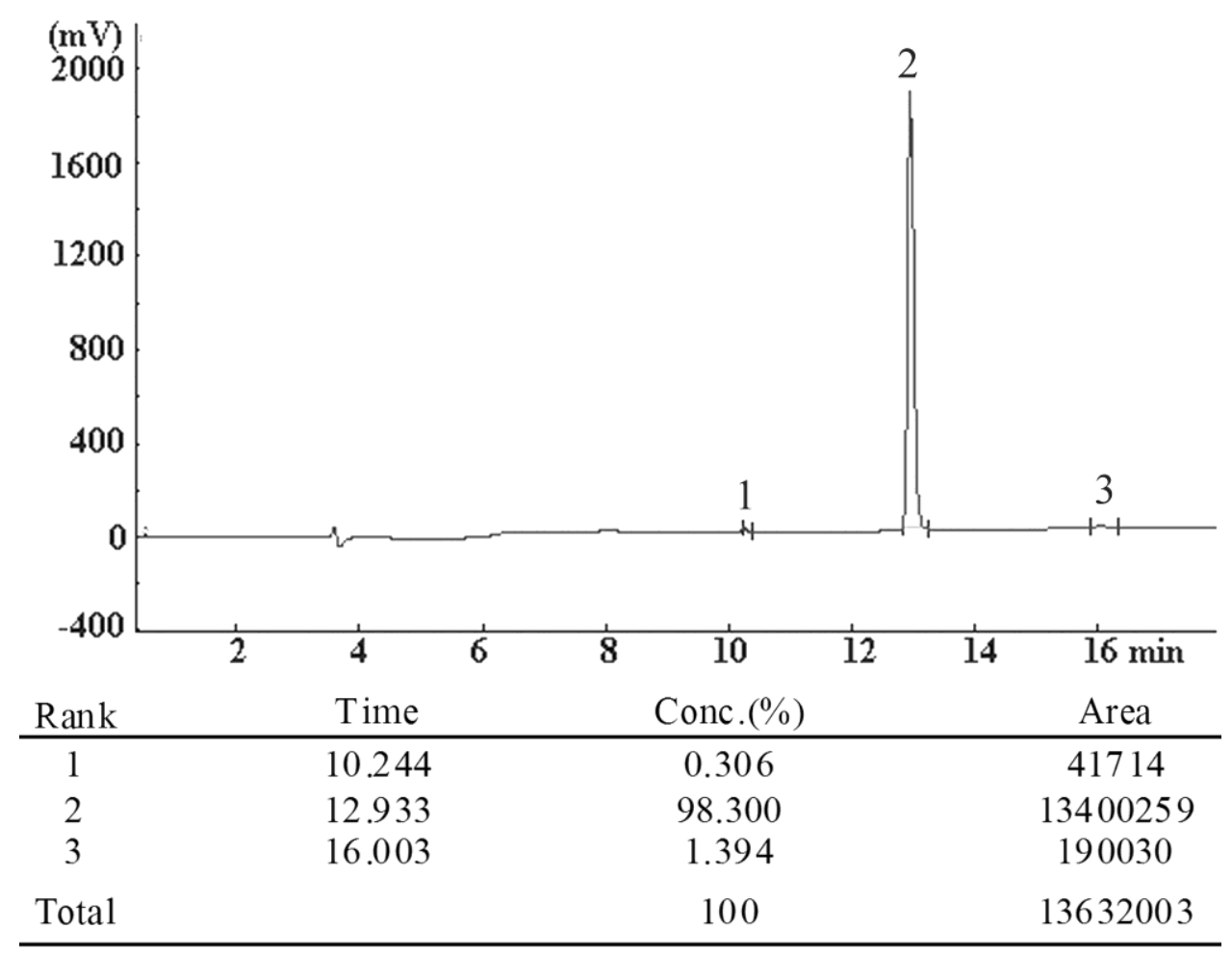


Fig. 3 Mass spectrometry results of the synthetic oligopeptide

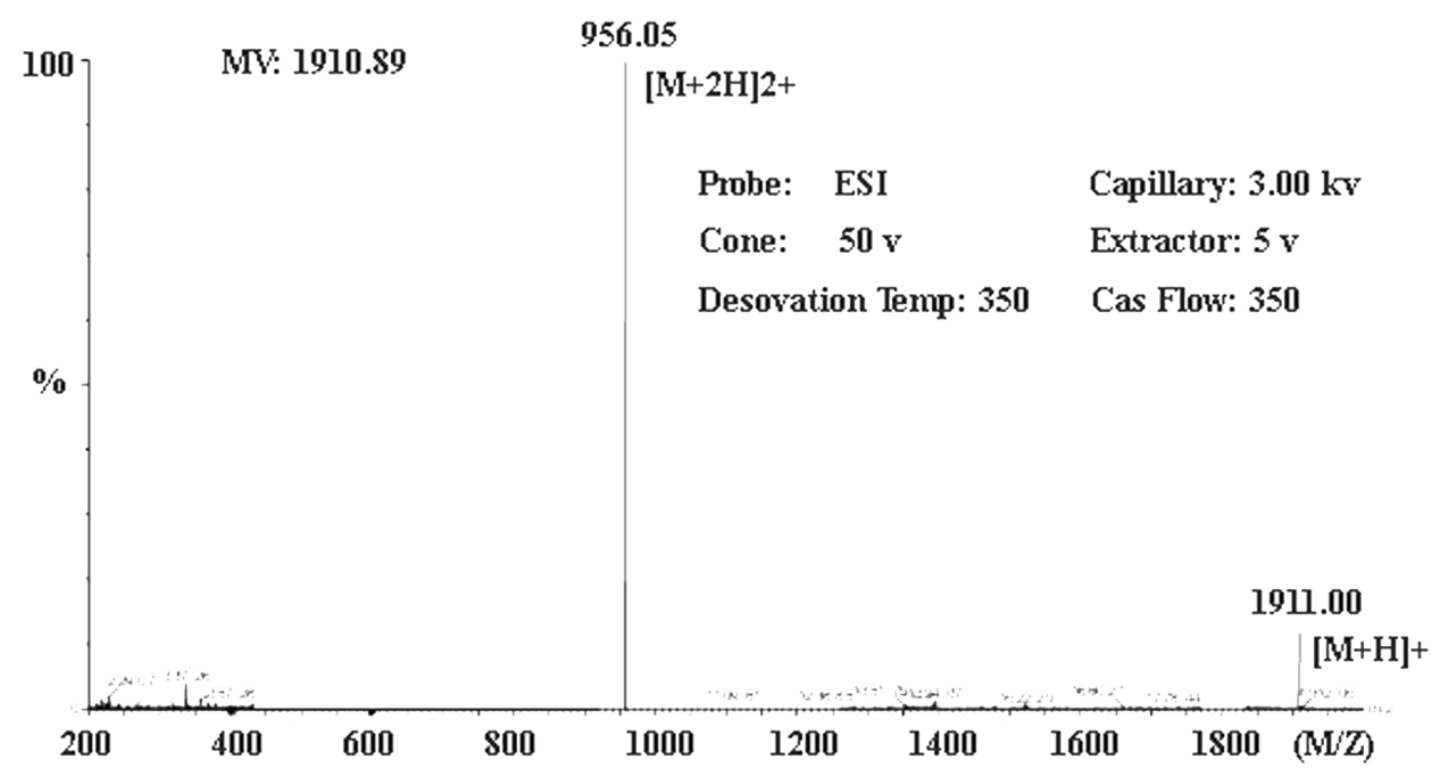

(The molecular weight of the synthetic oligopeptide is approximately $1,911 \mathrm{Da}$ )

Fig. 4 Fluorescent micrographs showing the application of the FITC-coupled oligopeptide (a), FITC-coupled IgG (b), and PBS (c) on dentine collagen

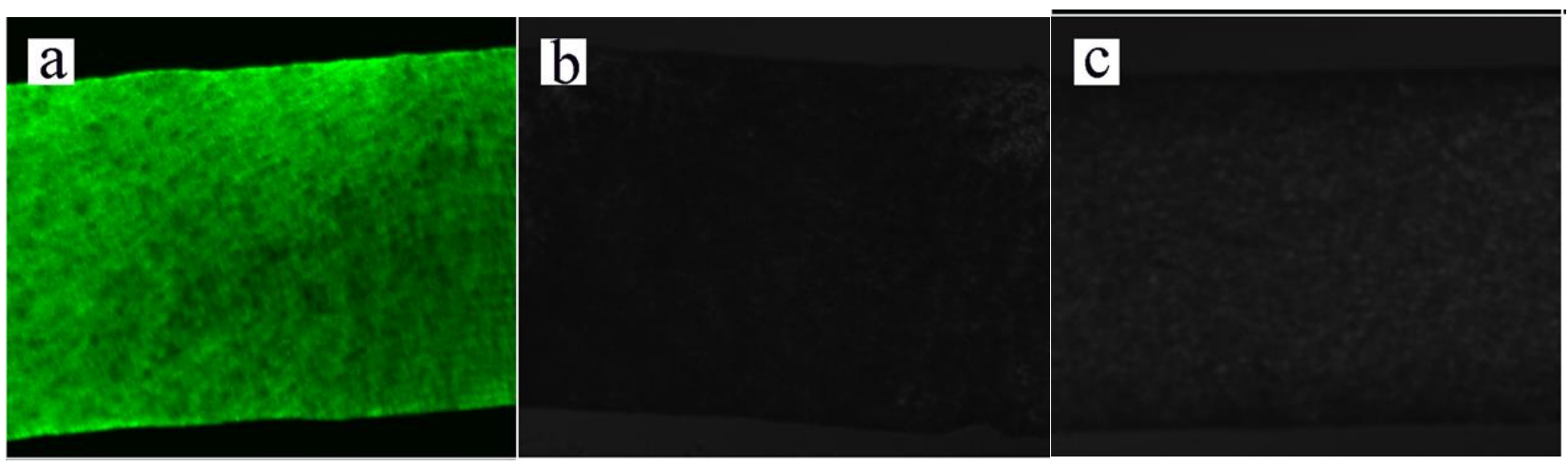

Micrograph (a) revealed fluorescence, which demonstrated the binding of the FITCcoupled oligopeptide to dentine collagen. Micrographs (b) and (c) showed no fluorescence, which illustrated no binding of the FITC-coupled IgG to collagen and no auto-fluorescence of dentine collagen, respectively. 
Fig.5 (a) SEM and (b) TEM image showing the hydroxyapatite precipitation after immersing the oligopeptide-coated TEM grids in calcium chloride and sodium hydrogen phosphate solutions, respectively, for 5 cycles. (b, inset) The electron diffraction pattern of the hydroxyapatite crystals. (c) SEM and (d) TEM image showing no precipitation in the control group (non-oligopeptide-coated TEM grids).
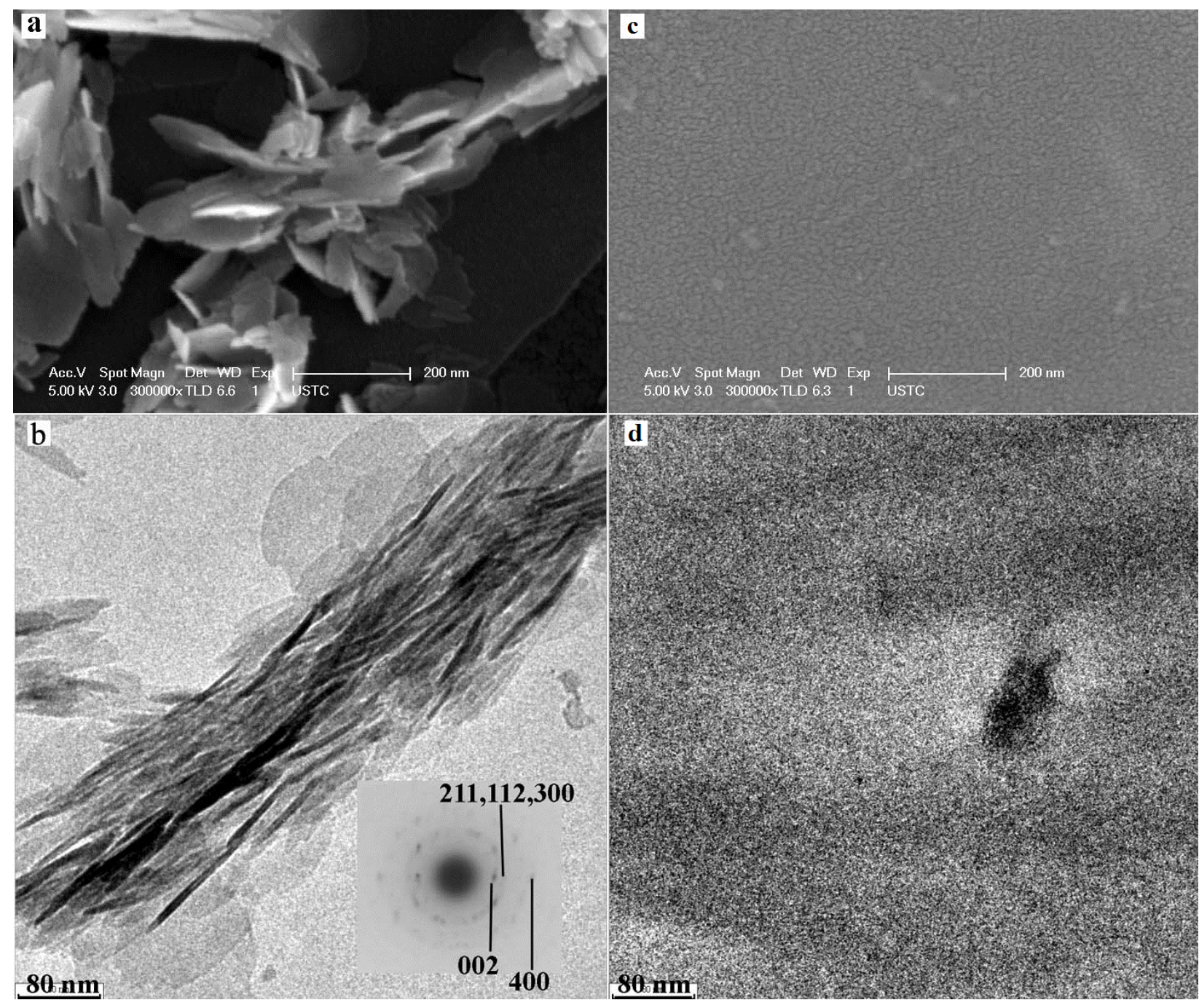
Fig. 6 The XRD pattern of the dentine collagen surface in different conditions

a: dentine collagen surface with synthetic oligopeptide coating after remineralization

b: dentine collagen surface without synthetic oligopeptide coating after remineralization

c: acid-etched dentine collagen surface before remineralization

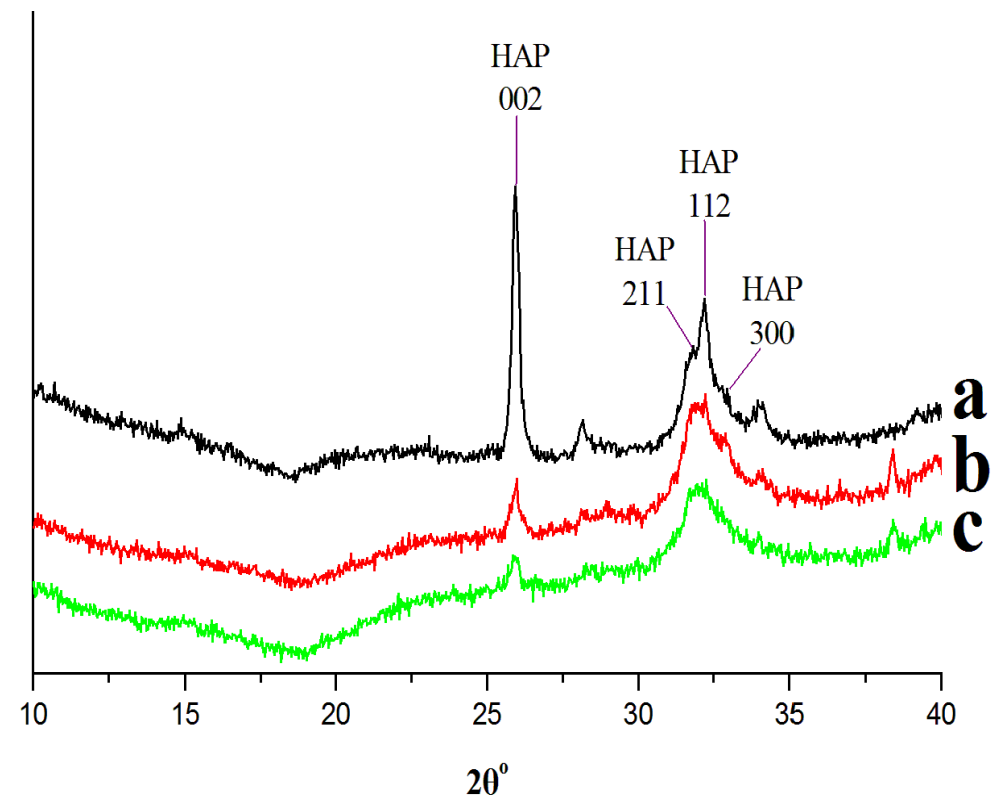

Fig. 7 DR-FTIR spectra of dentine collagen surface in different conditions

a- dentine collagen surface with synthetic oligopeptide coating after mineralization

b- dentine collagen surface without synthetic oligopeptide coating after mineralization c- acid-etched dentine collagen surface before mineralization

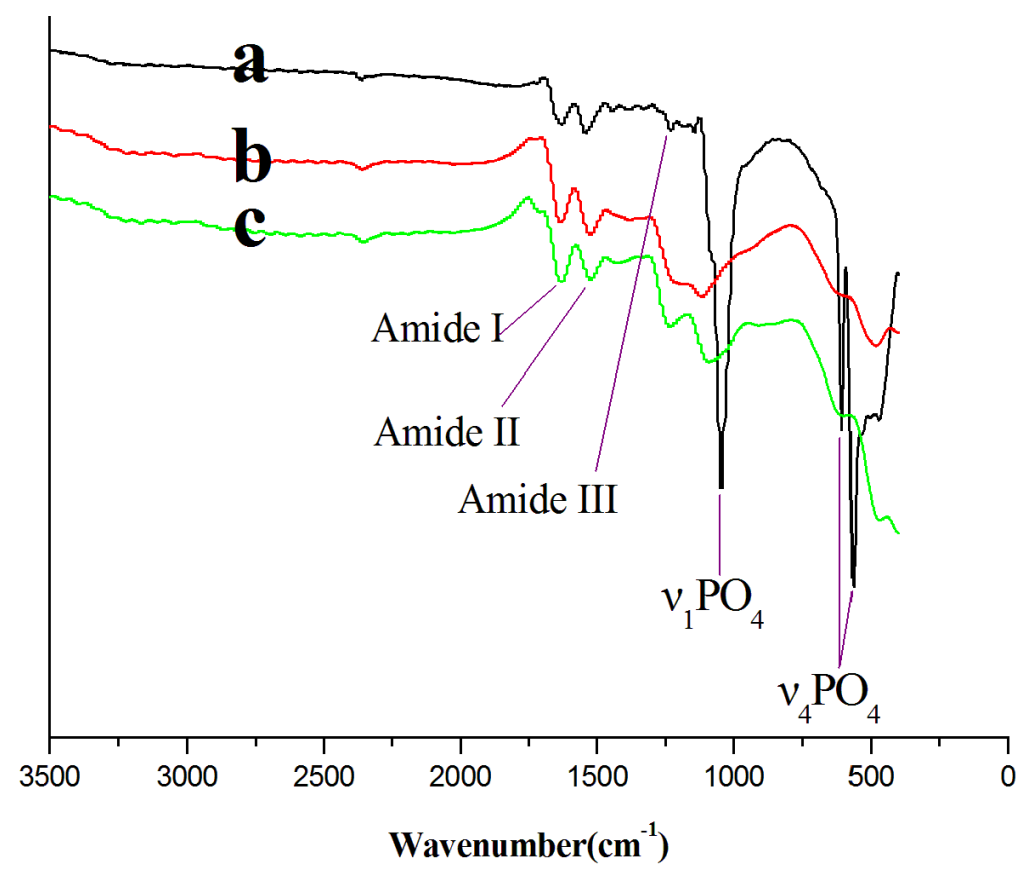


Fig. 8 SEM images of the oligopeptide-coated dentine surfaces after 48 hours of mineralization. (a) surface topography, (b to f) transverse sections. The white line in (b) shows the interface between the demineralized area (upper, showing the wide dentinal tubules after acid etching) and non-demineralized aears (below). (c) magnification of the demineralized areas after remineralization showing that the demineralized collagen matrix is calcified and the dentinal tubules are occluded. (d) magnification of subsurface areas of remineralized collagen matrix which have not reached to the density of natural dentine (e, f). (e) magnification of the deep areas of non-demineralized dentine showing the mineral crystals occlude dentinal tubules and the natural calcified dentine collagen matrix. (f) is the magnification of (e).

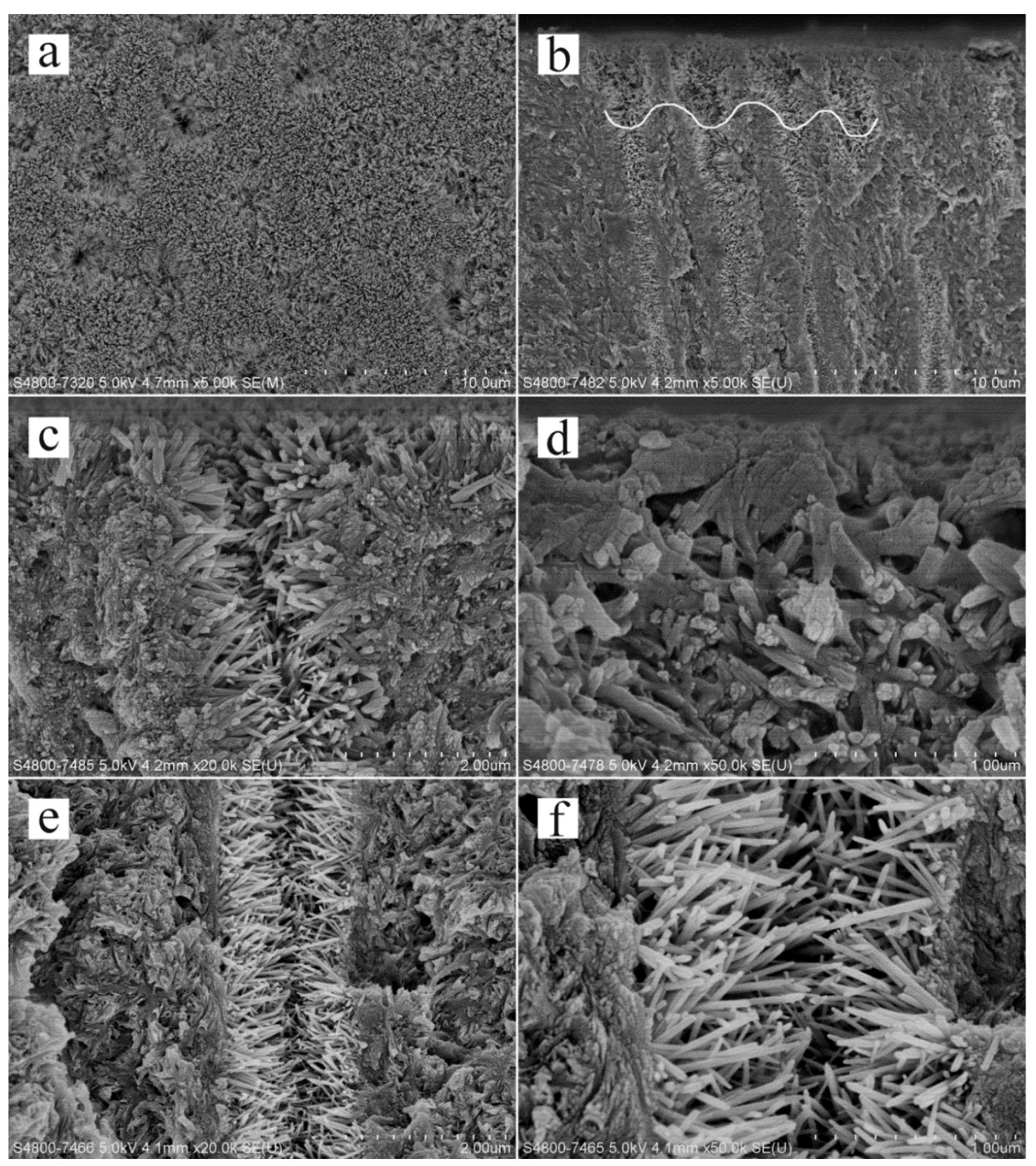


Fig. 9 SEM iamges of the dentine surface in the control group after 48 hours of mineralization. The images showed no HAP crystals on the dentine surface (a) and in the dentinal tubules after 48 hours (b).

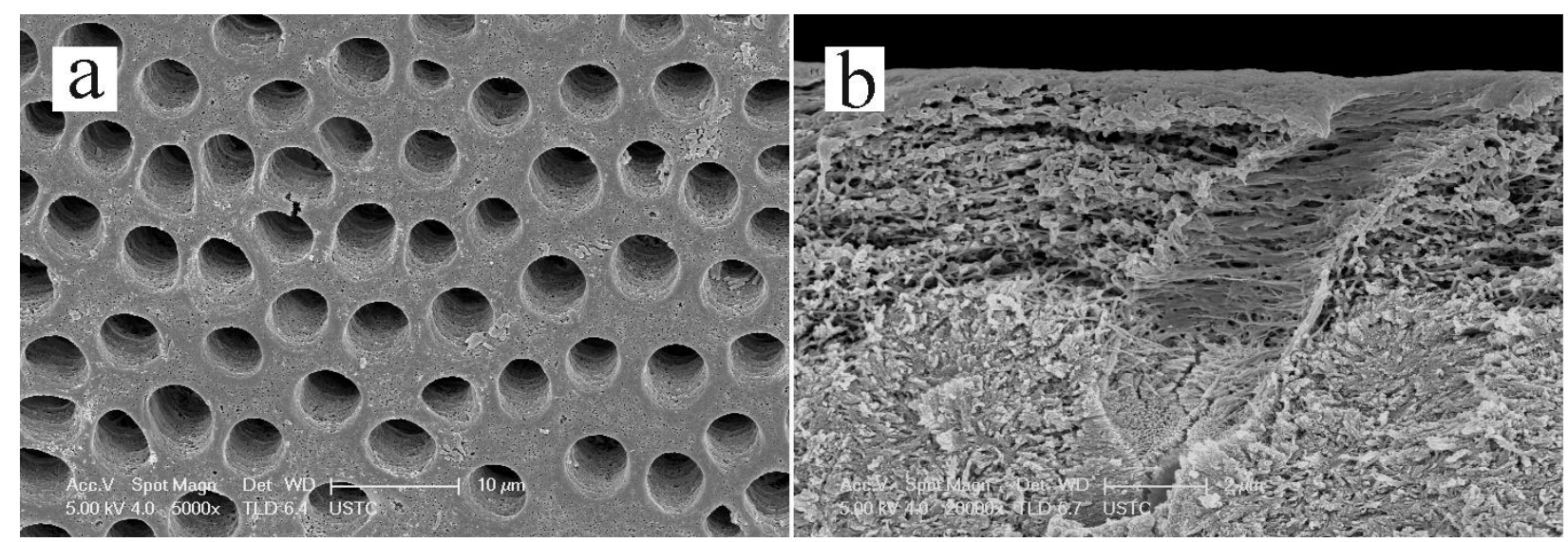

